\title{
Slow and steady progress in managing gynecologic cancer
}

\author{
Jane de Lartigue, $\mathrm{PhD}$
}

$\mathrm{S}$ low but steady progress has been made in the management of the major types of gynecologic malignancy, with particularly significant advancements in the treatment of the biggest killer, ovarian cancer. Here we describe how that progress has shaped the current treatment landscape and is forging a path forward.

\section{A therapeutic challenge}

More than 90,000 new cases of gynecologic malignancy are diagnosed in the United States each year, and about a third of patients will ultimately succumb to their disease. ${ }^{1}$ Five major tumor types make up this large and varied group of cancers: cervical, ovarian, endometrial, vaginal, and vulvar, each with unique biology, etiology, and pathology. ${ }^{2}$

Most cervical cancers are squamous cell carcinomas and are caused by infection with human papillomaviruses (HPVs), a group of more than 200 related viruses. Development of effective screening methods and prophylactic vaccination have driven a substantial reduction in the incidence of cervical cancer in developed countries, though it remains a major cause of mortality in developing countries.

Endometrial cancer is the most commonly diagnosed gynecologic malignancy, while ovarian cancer is the most lethal (Figure 1). Most ovarian cancers are epithelial in origin and tend to be grouped with fallopian tube and primary peritoneal cancers for treatment purposes, and cancers arising in the uterus are mostly adenocarcinomas. Improvements in incidence and mortality for these two cancer types have been more modest than for cervical cancer.

Vaginal and vulvar cancers are significantly rarer, accounting for around 8\% of gynecologic cancers. They are predominantly squamous cell carcinomas and, like cervical cancer, HPV is a major risk factor. The incidence and mortal- ity rates of these cancers have remained stubbornly stagnant in the past few decades. ${ }^{3-7}$

Until recently, the treatment of gynecologic cancers mainly involved a combination of surgery, chemotherapy, and radiation therapy. In general, these tumor types can be readily cured if they are diagnosed early, but once they reach more advanced stages, treatment options are limited and there is a substantial therapeutic niche. This is particularly true for ovarian cancer, in which a lack of symptoms early on in the disease course and a paucity of effective treatment options for more advanced stages has earned it the moniker of "silent killer."

Researchers have attempted to fill the therapeutic void with numerous different types of targeted therapies and immunotherapies, following the success of these types of drugs in other tumor types. Despite slow progress and a number of clinical disappointments, significant advances have been made in several of the major types of gynecologic cancer.

\section{Cutting off the blood supply}

Angiogenesis, the formation of new blood vessels

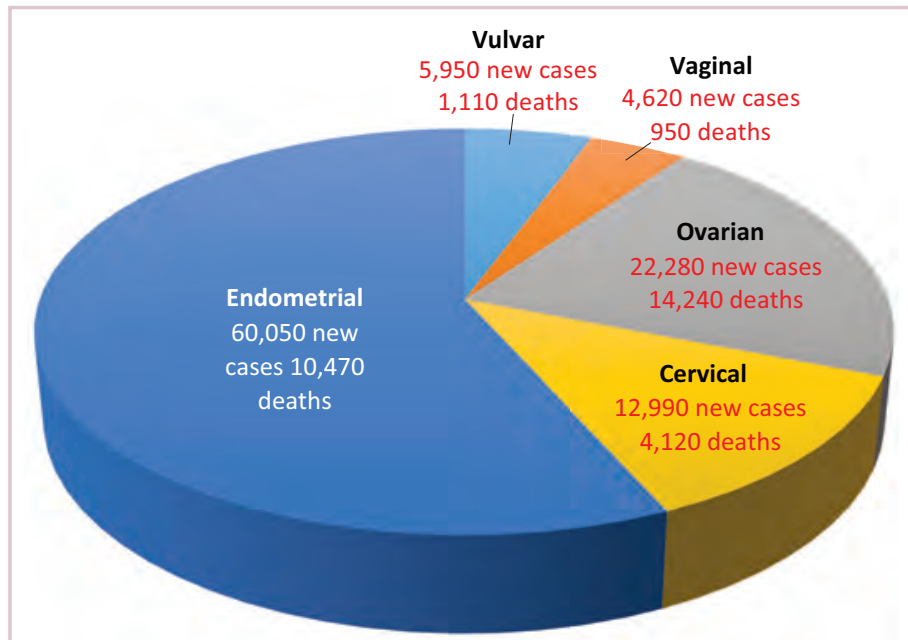

FIGURE 1 The incidence of the five main types of gynecologic cancer in the United States. The number of new cases and deaths are 2016 estimates from the American Cancer Society (www.cancer.org). 


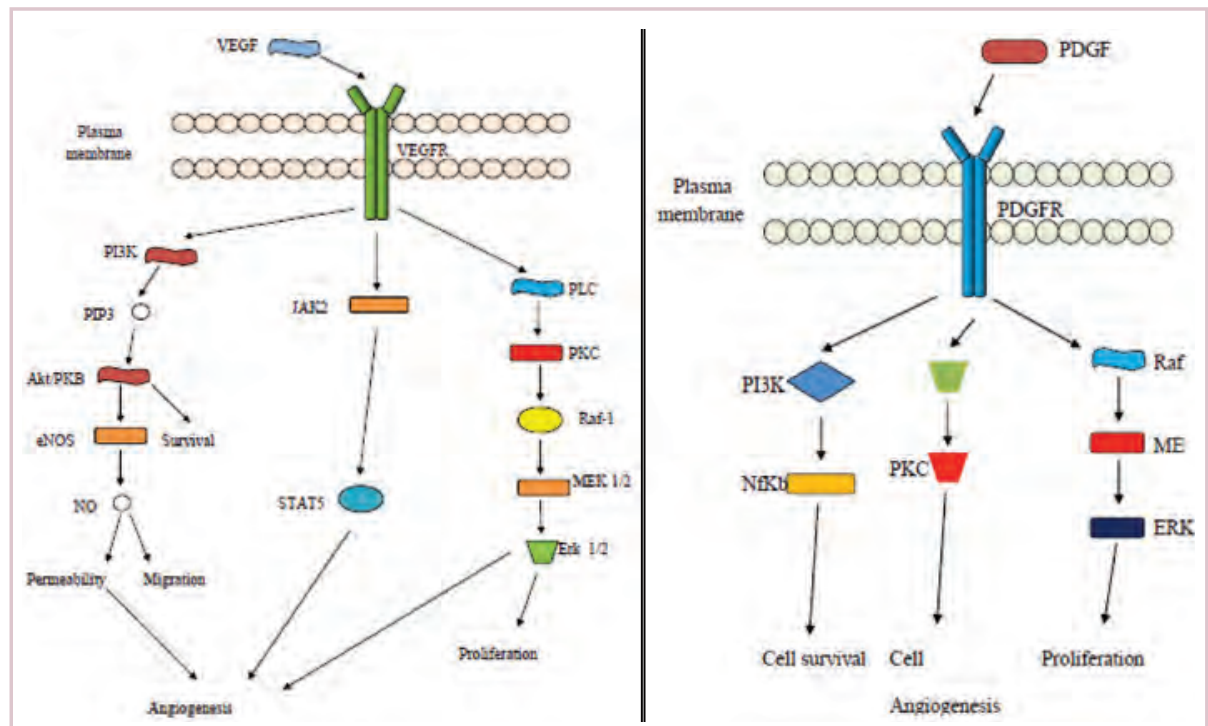

FIGURE 2 Several angiogenesis pathways have been shown to be important for the development of gynecologic cancers. Therapies targeting the central regulators of angiogenesis, the vascular endothelial growth factor (VEGF) and its receptor (VEGFR), as well as the platelet-derived growth factor receptor (PDGFR) pathway have been tested, with varying degrees of success. Reproduced with permission: Gavalas NG, Liontos M, Trachana S-P, et al. Angiogenesis-Related Pathways in the Pathogenesis of Ovarian Cancer. Int J Mol Sci. 2013;14:15885-15909.

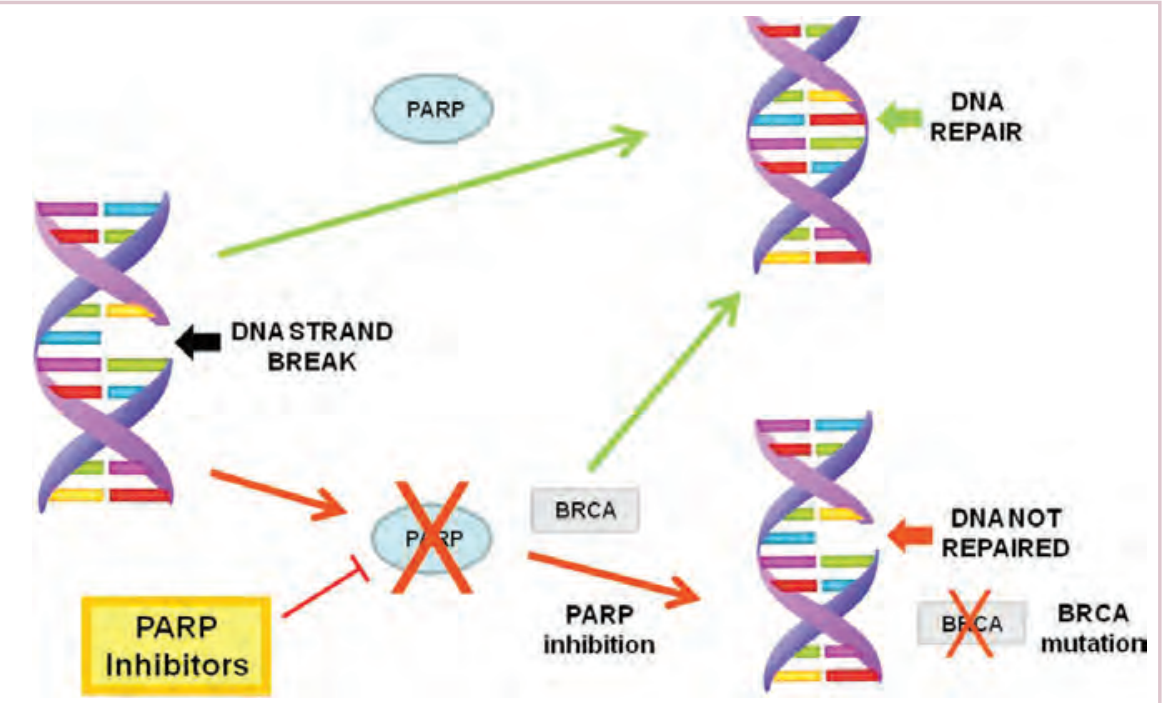

FIGURE 3 Poly(ADP-ribose) polymerase (PARP) inhibitors block the function of the PARP enzymes, which play a key role in the base excision repair pathway that repairs single-strand breaks (SSBs), a common form of DNA damage. In the presence of PARP inhibitors SSBs go unrepaired, leading to the formation of double-stranded breaks. DSBs can still be repaired by the homologous recombination (HR) pathway, in which the breast cancer susceptibility (BRCA) genes are involved, but in cancer cells that have defective BRCA genes or other faults in HR pathways, neither the SSB nor the resultant DSB can be repaired, which is a fatal combination for the cell. Reproduced with permission: Gibson SJ, Tewari KS, Monk BJ, Chase DM. Updates on drug discovery in ovarian cancer. Gynecol Oncol Res Pract. 2014;1:3.

from the pre-existing vasculature, plays a well-established role in tumor progression and is one of the "hallmarks" of cancer - an essential biological capability required for the transformation of a normal cell into a cancerous one. ${ }^{8}$
Under normal circumstances, there is a delicate balance between pro- and anti-angiogenic signals, which ensure angiogenesis is switched on only when required for normal physiological processes. In tumors, genetic alterations in the key angiogenic signaling pathways and the low-oxygen environment contribute to an angiogenic switch that pushes the balance towards pro-angiogenic signaling and allows the tumor to establish its own network of blood vessels to improve delivery of the oxygen and nutrients it needs to grow and to allow it to spread beyond its primary location. ${ }^{9}$

Drugs have been developed that target components of angiogenic signaling pathways, predominantly vascular endothelial growth factor (VEGF) and its receptor (VEGFR; Figure 2). These have proved to be effective in a number of different tumor types. In 2014, the VEGF-targeting monoclonal antibody, bevacizumab, became the first new therapy approved by the US Food and Drug Administration (FDA) for the treatment of ovarian cancer in almost a decade and the first in cervical cancer for more than 15 years.

Bevacizumab's approval for cervical cancer was based on the demonstration of improved overall survival (OS) in the phase 3 GOG 240 trial, in which a combination of bevacizumab and chemotherapy extended OS from 12.9 to 16.8 months, compared with chemotherapy alone. In ovarian cancer, although no OS advantage was observed, the addition of bevacizumab to non-platinum chemotherapy in platinum-resistant patients significantly improved progression-free survival (PFS) in the phase 3 AURELIA trial. $^{10,11}$

Numerous clinical trials of bevacizumab are ongoing, particularly in the setting of ovarian cancer, in which researchers are attempting to determine the optimal use of this drug, including duration, timing, and combination with other anti-cancer agents. Bevacizumab is also being tested in the setting of endometrial cancer in several different phase 1 and 2 clinical trials (Table, p. 370).

Anti-angiogenic therapies have expanded beyond beva- 
cizumab and the targeting of VEGF. The platelet-derived growth factor receptor (PDGFR) has also emerged as a key player in angiogenesis and several multitargeted tyrosine kinase inhibitors have been developed that block the activity of VEGFR and PDGFR and often several other important oncogenic receptors (Figure 2). Among them, cediranib, which targets all three VEGFRs, as well as PDGFR and c-KIT, has shown the most promise.

The results of the phase 3 ICON6 trial of cediranib in combination with chemotherapy in platinum-sensitive ovarian cancer were recently reported. A total of 486 women were randomly assigned to receive placebo and chemotherapy, followed by placebo-only maintenance (arm A), cediranib and chemotherapy, followed by chemotherapy-only maintenance (arm B), or cediranib and chemotherapy, followed by cediranib maintenance (arm C). Over a median follow-up of 19.5 months, the median PFS for the three groups was 8.7, 9.9, and 11 months, respectively. There was a trend toward improved OS, and though the data are not yet mature it offers the tantalizing possibility of the first anti-angiogenic drug to improve OS in ovarian cancer. $^{12}$

Cediranib continues to be evaluated in other phase 3 trials and has also received significant attention in combination with the poly(ADP-ribose) polymerase (PARP) inhibitor olaparib. In a phase 2 trial in women with recurrent platinum-sensitive ovarian cancer and mutations in the breast cancer susceptibility genes (BRCA1 and $B R C A 2)$, this combination almost doubled PFS compared with olaparib alone (9 vs 17.7 months). ${ }^{13}$ Phase 3 trials of this combination are underway in platinum-sensitive and -resistant patients (NCT02446600, NCT02502266).

The prospects for cediranib in cervical cancer are less clear. In a recently conducted phase 2 trial in combination with carboplatin-paclitaxel chemotherapy, PFS was improved in the cediranib arm, but no difference in OS was detected as yet. ${ }^{14}$

As an alternative to anti-angiogenic therapies, a newer class of drugs known as vascular disrupting agents, has been developed. Instead of preventing the formation of new blood vessels through angiogenesis, these drugs are designed to disrupt the existing tumor vasculature, causing it to collapse. An example is fosbretabulin, which is being studied in platinum-resistant ovarian cancer. A recently reported phase 2 trial demonstrated that fosbretabulin reduced the risk of disease progression by more than $30 \%$ in combination with bevacizumab, though there was an increase in grade 2 and higher hypertension. ${ }^{15}$

\section{Exploiting DNA damage}

Another exciting development for the treatment of gynecologic cancers is PARP inhibitors (Figure 3). These drugs target a family of enzymes that play an important role in repairing DNA damage that occurs following environmen- tal assaults or errors introduced during cellular replication. Cells have a number of different means of repairing DNA damage, which include base excision repair (BER), nucleotide excision repair (NER), homologous recombination (HR), and non-homologous end-joining (NHEJ). PARPs are critical to the BER pathway, binding to single-stranded breaks (SSBs) in the DNA and recruiting repair proteins to fix them.

PARP inhibitors block PARP activity, preventing SSB repair. These $\mathrm{SSB}$ can be repaired by alternative methods, unless there are additional failures in the DNA repair pathways, such as mutations in the $B R C A 1 / 2$ genes, as the proteins they encode are integral to the HR repair pathway. In this case, the SSBs induced by PARP inhibition go unrepaired and develop into lethal double-stranded breaks (DSBs).

PARP inhibition exploits the concept of synthetic lethality - the idea that two individual cellular defects, which the cell can cope with when they are present in isolation, when combined cause cell death. There are several routes to synthetic lethality with PARP inhibitors; they can be used as monotherapy in patients with mutations in DNA repair pathways, such as the aforementioned BRCA1/2 mutations, or they can be used in combination with other drugs that cause DNA damage. ${ }^{16-18}$

BRCA mutations are observed in $10 \%-15 \%$ of patients with ovarian cancer and therefore PARP inhibitors have also been the focus of clinical trials in this cancer type. ${ }^{19} \mathrm{In}$ December 2014, olaparib was granted accelerated approval for the treatment of advanced $B R C A$ mutation-positive ovarian cancer on the basis of response rates of more than $30 \%$ in a single-arm, phase 2 trial in 317 patients who received $400 \mathrm{mg}$ twice-daily oral olaparib. ${ }^{20}$

The idea of olaparib as maintenance therapy in patients who respond to their primary therapy, has also been explored. The FDA has so far declined to approve olaparib for this indication, despite demonstration of a robust PFS benefit, because of the lack of improvement in OS. The results of a third interim analysis of a phase 2 trial of olaparib maintenance therapy were reported at the 2016 meeting of the American Society of Clinical Oncology (ASCO) in June. Though it was just shy of achieving statistical significance, olaparib did confer an OS advantage, with a $27 \%$ reduction in the risk of death in the overall population and a $38 \%$ reduction in patients with $B R C A$ mutations. Fifteen percent of patients received olaparib for more than 5 years, suggesting that long-term olaparib maintenance therapy is a possibility. ${ }^{21}$

Several additional PARP inhibitors are following in olaparib's footsteps. The phase 2 ARIEL-2 and phase 3 ARIEL-3 trials for rucaparib are ongoing. Very positive results from ARIEL-2 were reported at ASCO in 2015; the ORR for rucaparib monotherapy in patients with $B R C A$-mutated relapsed ovarian cancer was $82 \%$ and in a 
TABLE A selection of therapies being evaluated in gynecologic malignancies

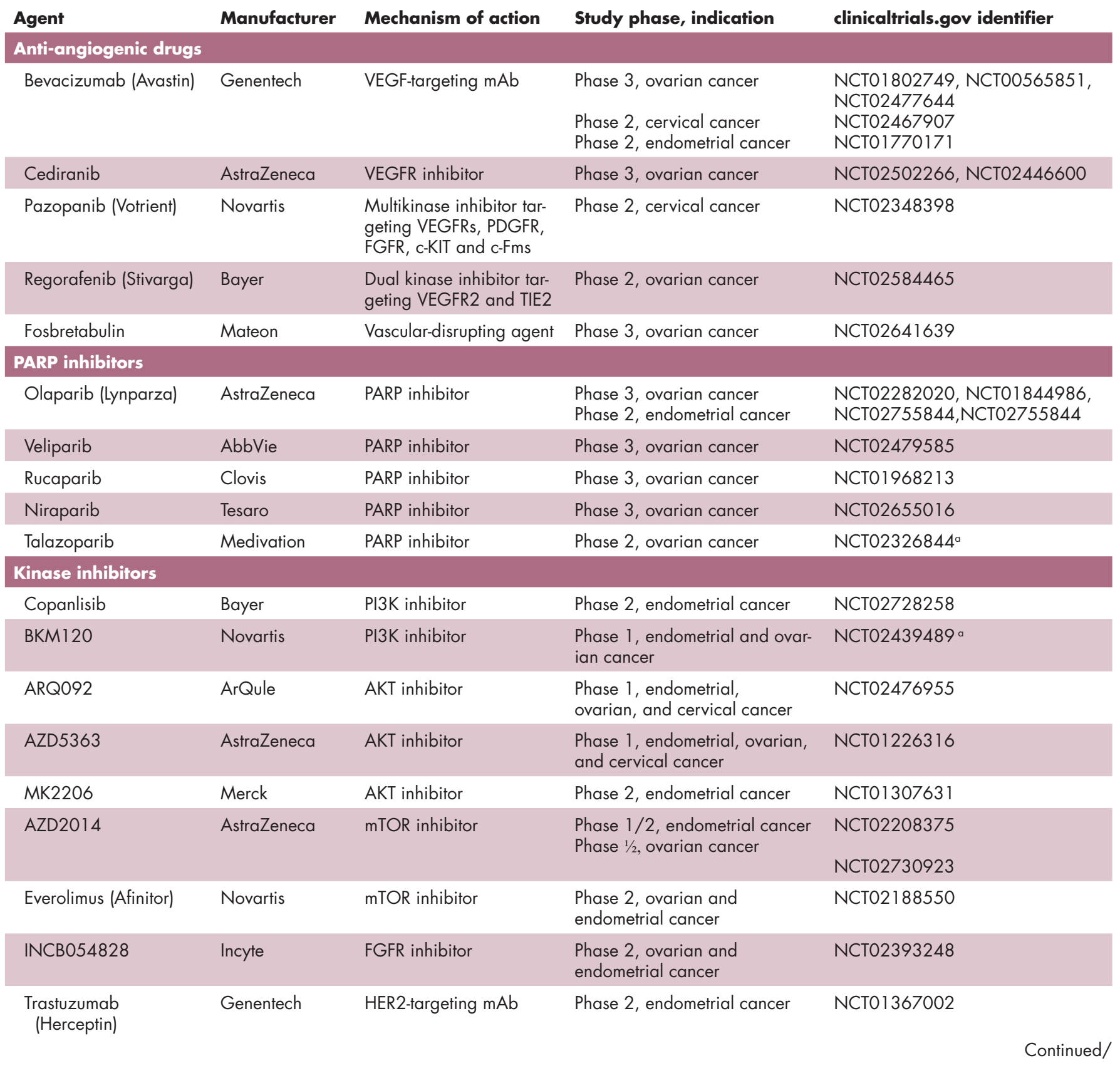

BRCA-like group defined by biomarkers associated with HR deficiencies it was $45 \% .{ }^{22}$

Niraparib is being evaluated in phase 3 trials as maintenance therapy in patients with HR-deficient advanced ovarian cancer. At the 2016 ASCO meeting, the results of part 1 of a two-part phase 1 study of niraparib in combination with bevacizumab in patients with recurrent platinum-sensitive ovarian cancer were presented. Among 12 patients, 1 had complete response, 4 partial response and 6 stable disease, for a disease control rate (DCR) of more than $90 \% .^{23}$
Talazoparib and veliparib both are undergoing phase 3 clinical testing in ovarian cancer. On the one hand, veliparib in combination with oral cyclophosphamide did not show improvement in ORR or PFS in a recently published phase 2 trial. ${ }^{24}$ Talazoparib, on the other hand, showed promise in phase 1 data presented at the 2016 annual meeting of the American Association of Cancer Research (AACR), with an ORR of $57 \%$ in 40 patients who received a combination of talazoparib and low-dose chemotherapy. ${ }^{25}$ 


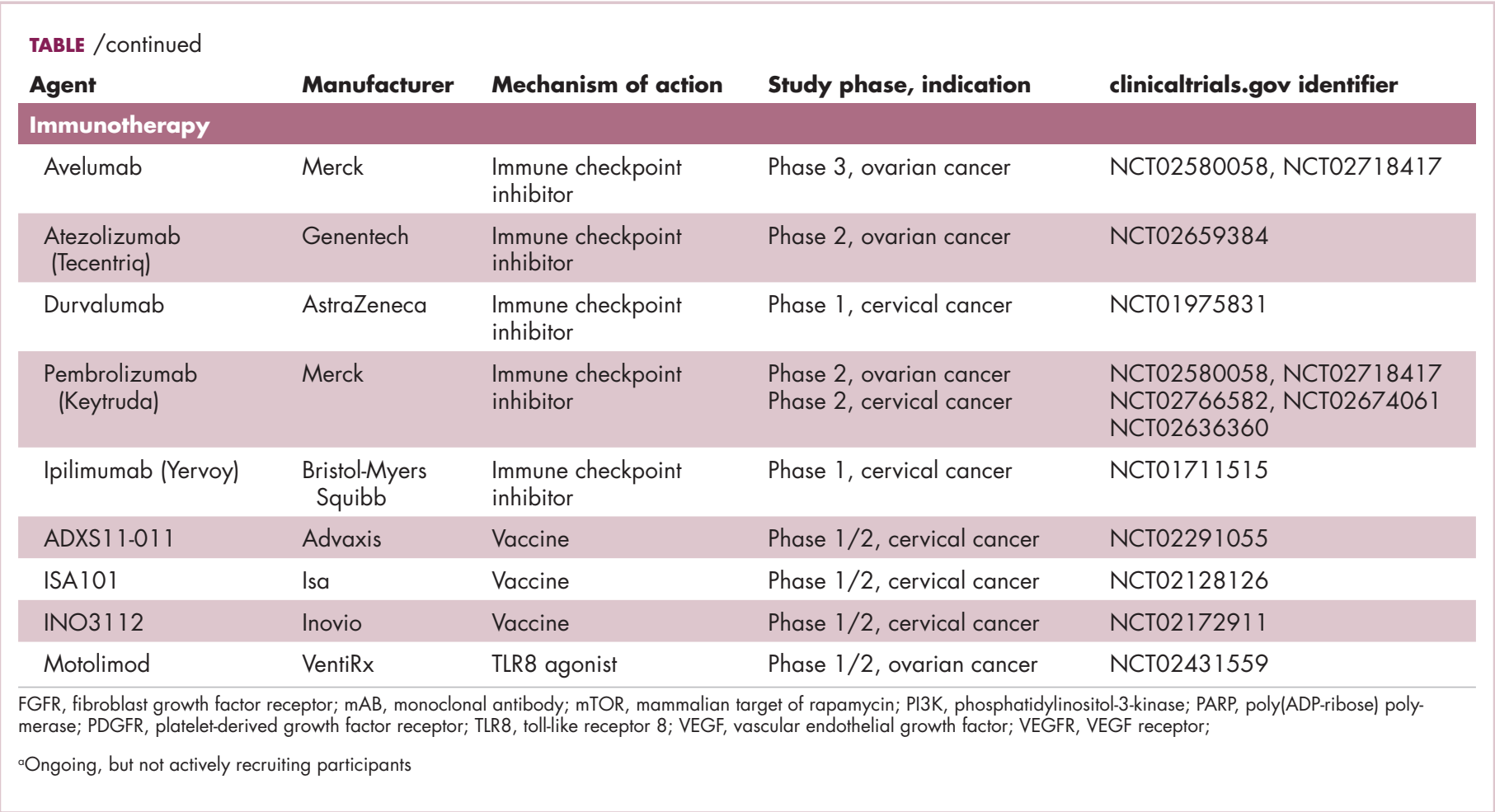

\section{Aiming at other targets}

The PI3K-AKT-mTOR pathway is one of the most frequently altered signaling pathways in several types of gynecologic malignancy, raising the possibility that inhibitors directed against component kinases of this pathway may be promising treatments. ${ }^{26}$ Several such drugs have been evaluated in endometrial and ovarian cancers, with varying degrees of success.

Everolimus has been particularly noteworthy in combination with the aromatase inhibitor, letrozole, in endometrial cancer. Some patients with endometrial cancer are responsive to hormonal manipulation with aromatase inhibitors and PI3K pathway activation may be one of the mechanisms underlying resistance to these drugs, thus presenting a rationale for combination therapy. A phase 2 study demonstrated a $32 \%$ ORR and $40 \%$ clinical benefit rate (CBR) for this combination and, in a presentation at the 2016 ASCO meeting, adding the anti-diabetic drug metformin, improved outcomes even further, with a CBR of more than $60 \% .^{27,28}$

The immunotherapy field has also gained some traction in the treatment of gynecologic malignancies and several different approaches have been tested. Therapeutic vaccination has been a particular focus in cervical cancer, particularly as the close association with HPV infection provides a useful target. Vaccination approaches have included simple peptide- and protein-based vaccines, as well as more complex cell-based vaccines. The most advanced in clinical development is ADXS11-011, a live vaccine designed to target the HPV E7 protein, currently in phase $1 / 2$ clinical trials. ${ }^{29}$

Immune checkpoint inhibitors that are designed to prevent immune cells from being switched off by cancer cells are the subject of intense study. Though clinical experience in gynecologic cancers remains limited, there are signs of activity from early-stage trials. The fully human monoclonal antibody targeting programmed cell death ligand 1 (PD-L1), avelumab, has progressed furthest in clinical development. Several phase 3 trials are ongoing and the results of the phase 1b JAVELIN trial were presented at ASCO 2016. The ORR was $9.7 \%$ based on 12 partial responses in patients with ovarian cancer, with a DCR of $54 \% .^{30}$ Preliminary results from a phase 1 trial of pembrolizumab (Keytruda) in squamous cell cervical carcinoma were also presented. The ORR was $12.5 \%$ with a median duration of 19 weeks. ${ }^{31}$

Compared with ovarian and cervical cancers, the role of targeted and immunotherapies is much less well studied in other gynecologic malignancies. Vulvar and vaginal cancers, in particular, remain very difficult to treat diseases with poor clinical outcomes, and their study is hindered also by the fact that they are rare. A better understanding of the molecular mechanisms of these gynecologic malignancies and endometrial cancer is needed, but the aberrations identified thus far suggest they could be amenable to existing therapies. However, where they have been included in phase 1 studies of solid tumors, only minimal clinical benefit has been attained. ${ }^{32}$ 


\section{References}

1. American Cancer Society. Cancer facts \& figures 2015. Atlanta: American Cancer Society; 2015.

2. Centers for Disease Control and Prevention. Gynecologic cancers. https://www.cdc.gov/cancer/gynecologic/. Last updated July 19, 2016. Accessed June 27, 2016.

3. Eifel PJ, Berek JS, Markman MA: Cancer of the cervix, vagina, and vulva. In: DeVita VT Jr, Lawrence TS, Rosenberg SA: Cancer: principles and practice of oncology. 9th ed. Philadelphia, Pa: Lippincott Williams \& Wilkins, 2011, pp 1311-1344.

4. Siegel R, Ma J, Zou Z, Jemal A. Cancer statistics, 2014. CA Cancer J Clin. 2014;64:9-29.

5. Tan W, Stehman FB, Carter RL. Mortality rates due to gynecologic cancers in New York state by demographic factors and proximity to a Gynecologic Oncology Group member treatment center: 1979-2001. Gynecol Oncol. 2009;114:346-352.

6. Howlader N, Noone AM, Krapcho M, et al. SEER Cancer Statistics Review, 1975-2012, National Cancer Institute. Bethesda, MD: April 2015.

7. Colombo N, Peiretti M, Parma G, et al. Newly diagnosed and relapsed epithelial ovarian carcinoma: ESMO Clinical Practice Guidelines for diagnosis, treatment and follow up. Ann Oncol. 2010;21(suppl 5):v23-v30.

8. Hanahan D, Weinberg RA. The hallmarks of cancer. Cell 2000;100:57-70.

9. Gavalas NG, Liontos M, Trachana S-P, et al. Angiogenesis-related pathways in the pathogenesis of ovarian cancer. Int J Mol Sci. 2013;14:15885-15909.

10. Tewari KS, Sill MW,Long HJ, et al. Improved survival with bevacizumab in advanced cervical cancer. N Engl J Med. 2014;370:734-743.

11. Pujade-Lauraine E, Hilpert F, Weber B, et al. Bevacizumab combined with chemotherapy for platinum-resistant recurrent ovarian cancer: the AURELIA open-label randomized phase III trial. J Clin Oncol. 2014;32:1302-1308.

12. Ledermann JA, Embleton AC, Raja F, et al. Cediranib in patients with relapsed platinum-sensitive ovarian cancer (ICON6): a randomized, double-blind, placebo-controlled phase 3 trial. Lancet 2016;387:1066-1074.

13. Liu JF, Barry WT, Birrer M, et al. Combination cediranib and olaparib versus olaparib alone for women with recurrent platinum-sensitive ovarian cancer: a randomised phase 2 study. Lancet Oncol. 2014;15:1207-1214.

14. Symonds RP, Gourley C, Davidson S, et al. Cerdiranib combined with carboplatin and paclitaxel in patients with metastatic or recurrent cervical cancer (CIRCCa): a randomised, double-blind, placebocontrolled phase 2 trial. Lancet Oncol. 2015;16:1515-1524.

15. Monk BJ, Sill MW, Walker JL, et al. Randomized phase II evaluation of bevacizumab versus bevacizumab plus fosbretabulin in recurrent ovarian, tubal, or peritoneal carcinoma: an NRG Oncology/ Gynecologic Oncology Group Study [published online ahead of print May 23, 2016.] J Clin Oncol. doi: 10.1200/JCO.2015.65.8153.

16. Benafif $S$ and Hall M. An update on PARP inhibitors for the treatment of cancer. Onco Targets Ther. 2015;8:519-528.

17. Liu FW and Tewari KS. New targeted agents in gynecologic cancers: synthetic lethality, homologous recombination deficiency and PARP inhibitors. Curr Treat Options Oncol. 2016;17:12.

18. Ledermann JA. PARP inhibitors in ovarian cancer. Ann Oncol.
2016;Suppl 1:i40-i44.

19. Alsop K, Fereday S, Meldrum C, deFazio A, Emmanuel C, George J, et al. BRCA mutation frequency and patterns of treatment response in BRCA mutation-positive women with ovarian cancer: a report from the Australian Ovarian Cancer Study Group. J Clin Oncol. 2012;30:2654-2663.

20. Kaufman B, Shapira-Frommer R, Schmutzler RK, et al. Olaparib monotherapy in patients with advanced cancer and a germline BRCA1/2 mutation. J Clin Oncol. 2015;33:244-250.

21. Ledermann J, Harter P, Gourley C, et al. Olaparib maintenance therapy in patients with platinum-sensitive relapsed serous ovarian cancer: a preplanned retrospective analysis of outcomes by BRCA status in a randomised phase 2 trial. Lancet Oncol. 2014;15:852-861.

22. McNeish IA, Oza AM, Coleman RL, et al. Results of ARIEL2: a phase 2 trial to prospectively identify ovarian cancer patients likely to respond to rucaparib using tumor genetic analysis. J Clin Oncol. 2015;33(suppl; abstract 5508).

23. Mirza RM, Mortensen CR, Christensen R, et al. A phase I study of bevacizumab in combination with niraparib in patients with platinum-sensitive epithelial ovarian cancer: The ENGOT-OV24/ AVANOVA1 trial. J Clin Oncol. 2016;34(suppl; abstr 5555).

24. Kummar S, Oza AM, Fleming GF, et al. Randomized trial of oral cyclophosphamide and veliparib in high-grade serous ovarian, primary peritoneal, or fallopian tube cancers, or BRCA-mutant ovarian cancer. Clin Cancer Res. 2015;21:1574-1582.

25. Wainberg ZA. Safety and efficacy results from a phase I dose-escalation trial of the PARP inhibitor talazoparib in combination with either temozolomide or irinotecan in patients with advanced malignancies. Presented at AACR annual meeting 2016; April 16-20, 2016; New Orleans, LA.

26. Salvesen HB, Werner HM, Krakstad C. PI3K pathway in gynecologic malignancies. Am Soc Clin Oncol Educ Book 2013. doi: 10.1200/EdBook_AM.2013.33.e218.

27. Slomovitz BM, Jiang Y, Yates MS, et al. Phase II study of everolimus and letrozole in patients with recurrent endometrial carcinoma [Published online before print January 26, 2015]. J Clin Oncol. doi: 10.1200/JCO.2014.58.3401.

28. Soliman PT, Westin SN, Iglesias DA, et al. Phase II study of everolimus, letrozole, and metformin in women with advanced/recurrent endometrial cancer. J Clin Oncol. 2016;34(suppl; abstract 5506).

29. Cohen EEW, Moore KN, Slomowitz BM, et al. Phase I/II study of ADXS11-011 or MEDI4736 immunotherapies alone and in combination, in patients with recurrent/metastatic cervical or human papillomavirus (HPV)-positive head and neck cancer. J Immunother Cancer. 2015;3(suppl 2):P147.

30. Disis ML, Patel MR, Pant S, et al. Avelumab (MSC0010718C; antiPD-L1) in patients with recurrent/refractory ovarian cancer from the JAVELIN Solid Tumor phase Ib trial: safety and clinical activity. J Clin Oncol. 2017;34(suppl; abstract 5533).

31. Frenel J-S, Le Tourneau C, O’Neil BH, et al. Pembrolizumab in patients with advanced cervical squamous cell cancer: preliminary results from the phase Ib KEYNOTE-028 study. J Clin Oncol. 2016;34(suppl; abstract 5515).

32. Fu S, Shi N, Wheler J, et al. Characteristics and outcomes for patients with advanced vaginal or vulvar cancer referred to a phase I clinical trials program: the MD Anderson cancer center experience. Gyn Oncol Res Pract. 2015;2:10-16. 